\title{
Potential-modulated Electrocapacitive Properties of Soft Microstructured Polypyrrole
}

\author{
Mikhail Vagin, Rodtichoti Wannapob, Yu Liu and Wing Cheung Mak \\ Journal Article
}

\section{Tweet}

N.B.: When citing this work, cite the original article.

Original Publication:

Mikhail Vagin, Rodtichoti Wannapob, Yu Liu and Wing Cheung Mak, Potential-modulated Electrocapacitive Properties of Soft Microstructured Polypyrrole, Electroanalysis, 2017. 29(1), pp.203-207.

http://dx.doi.org/10.1002/elan.201600261

Copyright: Wiley: 12 months

http://eu.wiley.com/WileyCDA/

Postprint available at: Linköping University Electronic Press

http://urn.kb.se/resolve?urn=urn:nbn:se:liu:diva-136077

H.U UNNGERES 


\title{
Potential-modulated electrocapacitive properties of soft microstructured polypyrrole
}

\author{
Mikhail Yu. Vagin ${ }^{a, b, * *}$, Rodtichoti Wannapob ${ }^{a, c, \neq}$, Yu Liu ${ }^{a, d}$, Wing Cheung Mak ${ }^{a *}$ \\ a Biosensors and Bioelectronics Centre, Department of Physics, Chemistry and Biology, Linköping University, Linköping, \\ Sweden; Tel: +4613286921 \\ *e-mails: mikva@ifm.liu.se, mamak@ifm.liu.se; ‡ These authors contributed equally. \\ baboratory of Organic Electronics, Department of Science and Technology, Linköping University, Norrköping, Sweden \\ c Department of Chemistry, Faculty of Science, Prince of Songkla University, Hat Yai, Songkla, 90112, Thailand \\ d College of Life and Science, Sichuan Agricultural University, Yaan 625014, People’s Republic of China.
}

Received: ((will be filled in by the editorial staff))

Accepted: ((will be filled in by the editorial staff))

\begin{abstract}
Microstructured materials are becoming important for high performance electrochemical device especially for energy storage due to their advantageous diffusion and flux properties. Utilizing a rationally designed hollow structured polypyrrole microparticles (PPyMPs) with controllable wall thicknesses of 110 to $340 \mathrm{~nm}$, we observed a significant morphological effect on electrocapacitive kinetics of the PPyMPs modulated by the voltammetric potential window and scan rate. The thinhollow architecture of PPyMPs revealed significant enhancement of charge storage performance (up to 447\%), high retention at high scan rate and faster charge/discharge kinetics compared to the thick-hollow PPyMPs due to the larger accessible surface area and decrease of diffusion length. These findings demonstrated the electrocapacitive kinetics performance of microstructured soft materials related to morphological effect modulated by operational conditions. Our study provides new insight on electrochemistry of soft electrode materials with controlled nanostructured morphology for understanding the mechanism of charge insertion and mass diffusion for the future development of high performance porous electrode material.
\end{abstract}

Keywords: Polypyrrole, Hollow microparticles, Potential-modulation, Scan rate, Charge storage

DOI: 10.1002/elan.((will be filled in by the editorial staff))

Considerable research effort has been put to the energy storage devices operating by means of electrochemical processes. Conducing polymers as a materials available for electrical charge storage due to the bulk redox reactions, combine a variety of exceptional properties such as electrical conductivity that cover the whole insulatorsemiconductor-metal range, reversible doping/de-doping processes, ionic conductivity, mechanical flexibility, lightness, biocompatibility and cost advantages [1].

Main Processable conducting polymers allow the device fabrication by simple deposition onto various substrates. Colloidal chemistry enables to fabricate polymer materials in the form of microparticles for development of solutionprocessable materials for direct fabrication of organic electrode interfaces. At the electrode interfaces, the whole electrochemical processes yielding the electrical charge storage inside conducting polymers involve simultaneous electrical conduction and ionic diffusion, which must be established at multiphase boundaries at the device operating conditions. Therefore, the morphology and structural organization of the electrode material is vital for enhanced device performance by facilitating the electrocapacitive kinetics and mass diffusion processes [2]. One of the possible ways towards this aim is to create multi-level hierarchical interfaces using microparticles with desired micro-/nanoscale morphology as building blocks that assembled into a macrodimensional structure. The hollow structured morphology is advantageous due to the thin wall morphology and double sided interfaces yielding faster charge/discharge kinetics and higher capacitance [3]. There are only few approaches to fabricate hollow structured conducting polymer microparticles includes the formation of the polymer shells on a template beads followed by the template dissolution [4] or the template-free polymerization micellar microenvironment [5].

Recently, we have reported the hard template synthesis of hollow structured polypyrrole microparticles (PPyMPs) with controllable wall thicknesses [6] and variety of functionalities [7]. The effect of soft morphology on the 


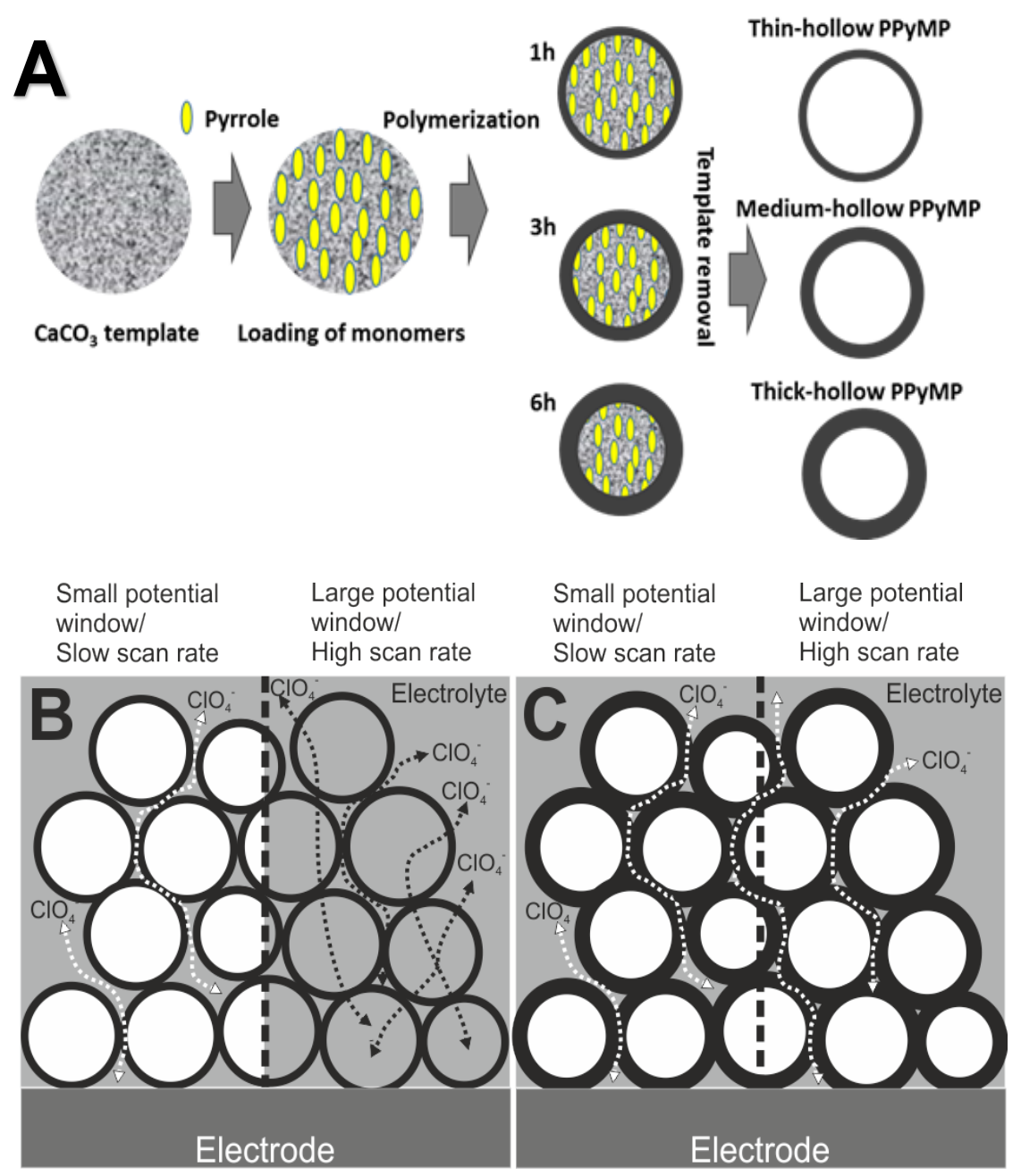

Scheme 1. (A) The hard template synthesis of PPyMPs of different scale wall thicknesses; the modulation of the material accessibility at films fabricated from thin- (B) and thick-hollow (C) microparticles

electrocapacitive properties of water-processable conducting material was systematically studied.

Here we report the effect of potential-modulated accessibility of soft electrode material (Scheme 1). The variations in the scan rate and potential window utilized for the electrocapacitive properties evaluation of films formed by microparticles with different wall thickness led to significantly different kinetics of charge storage.

PPyMPs with tunable wall thickness were fabricated with $\mathrm{CaCO}_{3}$ template-assisted time dependent polymerization technique (Scheme 1A). By controlling the polymerization time ranging from 1, 3 and 6 hour(s), we were able to obtain PPyMPs with controlled wall thickness (Fig. 1A-C). PPyMPs obtained from 1 and 3 hour(s) polymerization appeared capsule-like morphology with a slightly transparency center region, while PPyMPs obtained from 6 hours polymerization appeared darker in color resulted from the increase of capsule thickness. Resulting from the advantage of the template synthesis, all the synthesized PPyMPs were fairly homogenous with narrow size distribution of diameter of $2.6 \pm 0.4 \mu \mathrm{m}$. The homogenous diameter of the PPyMPs is critical which allows the study on the net effect of the wall thickness of soft materials contributing to ion diffusion/penetration and multiphase boundaries reactions. SEM images further showing the morphology of the deposited PPyMPs film composed of assembled microparticles (Fig. 1D-F). From the SEM images of the broken and collapsed PPyMPs, the wall thickness of the thin- and medium-hollow PPyMPs were estimated to be approximately $110 \pm 12 \mathrm{~nm}$ and 212 $\pm 22 \mathrm{~nm}$, respectively $(\mathrm{n}=10$; measurements were taken from different locations). The capsule thickness of the thick-hollow PPyMPs was estimated on the basis of the polymerization kinetics to be $340 \mathrm{~nm}$ (Fig. 1G).

The effect of the structural organization of the material on energy storage characteristics was investigated by voltammetry at GCE modified with same loading mass (50 $\mu \mathrm{g}$ calculated from the dry weight) of PPyMPs with 

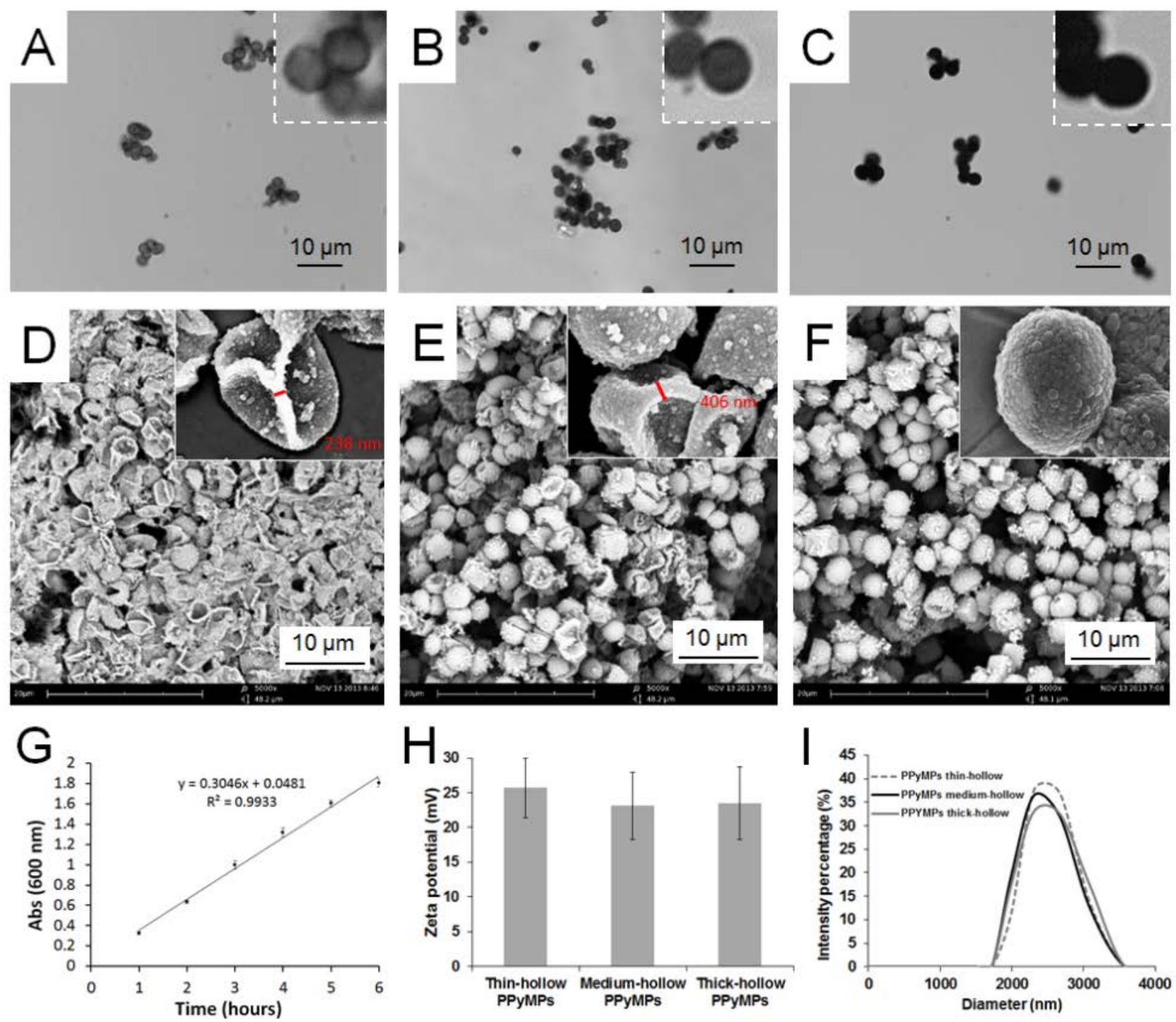

Fig. 1. $(A-C)$ Phase contrast images and (D - F) SEM images of PPyMPs fabricated with polymerization time of 1 , 3 and 6 hours, respectively; insets shows the high magnification images of different PPyMPs. (G) Polymerization kinetics of PPyMPs measured by spectrophotometry at $600 \mathrm{~nm}$. (H) Zeta potentials and (I) size distribution curves of thin-, medium and thick-hollow PPyMPs, respectively.

different morphologies, while having similar chemical composition, surface zeta potential and physical diameter (Fig. 1H-I)).The deposited PPyMPs layers were cured at $60{ }^{\circ} \mathrm{C}$ for 30 minutes to promote assembly of PPyMPs to create hierarchical film structure.

Ensuring the measurement stability the voltammetric measurements have been carried out on equilibrated films. The voltammograms obtained in narrow potential window showed a symmetric box-shape typical for electrochemical supercapacitor behavior (Fig. 2A-C). The apparent capacitance values were 22.9, 10.7 and $4.9 \mathrm{~F} \mathrm{~g}^{-1}$ for thin, medium- and thick-hollow PPyMPs, respectively. The reproducibility of obtained voltammetric responses was within 20\%. The thin-hollow architecture of PPyMPs revealed significant enhancement of charge storage performance (up to 447\%), high retention at high scan rate and faster charge/discharge kinetics compared to the thickhollow PPyMPs due to the larger accessible surface area and decrease of diffusion length [8]. The surface-to-mass ratios $\left(\mathrm{m}^{2} \mathrm{~g}^{-1}\right)$ of the thin-, medium- and thick-hollow PPyMPs including the surface area of inner cavities were calculated with the values of $14.9,6.7$ and $4.93 \mathrm{~m}^{2} \mathrm{~g}^{-1}$, respectively. The calculation is based on the experimental average diameter of PPyMPs of $2.6 \mu \mathrm{m}$, wall thickness of thin $(110 \mathrm{~nm})$, medium $(212 \mathrm{~nm})$ and thick-hollow (340 $\mathrm{nm})$ PPyMPs, density of PPy of $1.54 \mathrm{~g} \mathrm{~cm}^{-3}$ while assuming the microparticles are prefect smooth spheres. This indicates the thin-hollow morphology revealed a larger accessible surface for interfacial ions insertion compared with thick-hollow structure. As the same time, 

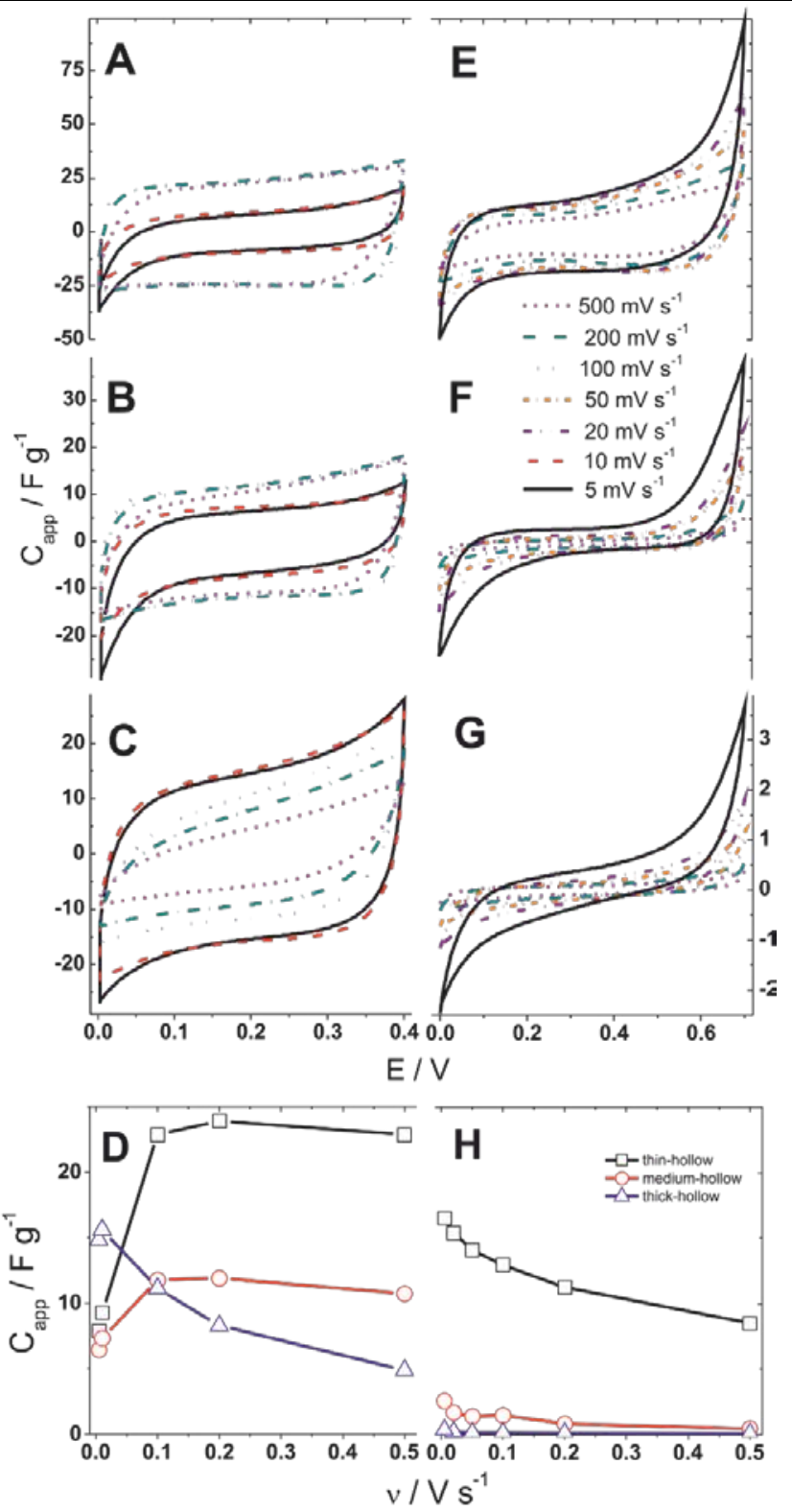

Fig. 2. Voltammetric characterization of energy storage properties of PPyMPs. Cyclic voltammograms normalized by the scan rate and mass of active material were obtained in narrow (A, B and $\mathrm{C}$ ) and wide (E, F, and $\mathrm{G}$ ) potential windows at electrodes modified with thin- (A and $\mathrm{E}$ ), medium- (B and $\mathrm{F}$ ) and thick-hollow PPyMPs (C and G; scan rates: 5, 10, 20, 50, 100, 200 and $500 \mathrm{mVs}^{-1}$ (black, red, purple, orange, blue, dark cyan and magenta respectively)); the dependences of the apparent capacitance on the scan rate obtained in narrow (D, at $200 \mathrm{mV}$ ) and wide $(\mathrm{H}$, at $300 \mathrm{mV})$ potential windows at electrodes modified with thin-, medium- and thick-hollow PPyMPs (black, red and blue respectively); aqueous electrolyte (0.05 $\left.\mathrm{M} \mathrm{LiClO}_{4}\right)$.

the thinner capsule wall thickness results in a decrease of the diffusion length providing the enhancement of the charge storage performance. Both thin- and medium- hollow PPyMPs revealed anomalous increases of capacitance with increase of scan rate (Fig. 2D) testifying the increase of material accessibility, which is rarely observed at the films of pseudocapacitive nanostructured electrode materials [9]. The higher rates of voltammetric potential ramp create stronger gradients of concentrations, which might open the access to the polymer/solution interface at the inner cavities inaccessible at slower scan rates (Scheme 1B).

Being equilibrated by the same procedure, PPyMP filmmodified electrodes showed the significant difference in the electrocapacitive behavior observed with cyclic voltammetry in the wider potential window (Fig. 2E-G). The currents obtained at the wider potential window are smaller than observed at the narrow window, which might illustrate the overoxidation of PPy at high potentials [10]. The wide potential window measurements showed the monotonous decrease of the apparent capacitance with the scan rate increase (Fig. $2 \mathrm{H}$ ), which is consistent with the majority of reports for porous electrode materials and illustrates the lower accessibility of the porous material at the faster potential ramp. However, consistent with the measurements at the narrow potential window, the thinhollow PPyMPs showed less distorted box-shaped response in comparison with the medium- and thickhollow attributed with significantly larger average values of apparent capacitance illustrating an morphological effect.

The values of specific capacitance estimated for hollowstructured drop-casted PPyMP films by cyclic voltammetry are in close agreement with the reported electrocapacitive characteristics of PPy-based composites [11]. To the best of our knowledge, the non-monotonous scan rate dependence of the specific capacitance for PPy, which illustrates the open structure of polymer, is reported for the first time. This leads to the higher retention of specific capacitance at high scan rates in comparison with the reported systems [11b, 11d], opening the possibility for the faster device operation.

The charge storage mechanisms have been studied by voltammetry on soft nanostructured conducting polymer with controlled wall thickness between 110 to $340 \mathrm{~nm}$. The systematic effect of microscale morphology yielded in dissimilar accessibility of soft electrode material to counterion insertion modulated by the scan rates or by potential window. The employment of hollow microscale architecture of porous electrode material allows the observation of non-monotonous dependence of the specific capacitance on the scan rate illustrating the possibility of electrocapacitive performance optimization by the rate of the potential ramp. These results demonstrated the electrocapacitive kinetics performance of conductive polymer materials is highly sensitive to the diffusion length (i.e. the wall thickness) at nanoscale to sub-microscale. 
Experimental

Calcium chloride, copper (II) perchlorate, pyrrole, sodium carbonate, ethylenediaminetetracetic acid (EDTA), lithium perchlorate, ethanol and 2-propanol were purchased from Sigma-Aldrich (St. Louis, MO, USA) and were used as received. Pyrrole (99\%) was received from Sigma-Aldrich and purified before use by passing through a neutral column of alumina $(0.05 \mu \mathrm{m})$ to obtain a colourless liquid. Water was purified using Milli-Q water purification system.

The polypyrrole microparticles (PPyMPs) with tunable morphology were prepared with time dependent template polymerization. In brief, $1 \mathrm{~mL}$ of $\mathrm{CaCl}_{2}(0.5 \mathrm{M})$ and $1 \mathrm{~mL}$ of $\mathrm{Na}_{2} \mathrm{CO}_{3}(0.5 \mathrm{M})$ were rapidly mixed at $600 \mathrm{rpm}$ and kept under stirring for 30 second to produce calcium carbonate $\left(\mathrm{CaCO}_{3}\right)$ microparticles as a removable template. The $\mathrm{CaCO}_{3}$ microparticles were washed consequently twice with water, ethanol and 2-propanol by centrifugation (2000 rpm., $1 \mathrm{~min}$ ) and re-dispersion cycles. The purified pyrrole as a monomer was loaded by mixing with $\mathrm{CaCO}_{3}$ microparticles followed by incubation for 30 min. The pyrrole-loaded $\mathrm{CaCO}_{3}$ microparticles were centrifuged (2000 rpm, $1 \mathrm{~min}$ ) and supernatant was discarded to remove the un-absorbed monomer. To carry out the polymerization, the resulting pyrrole-loaded $\mathrm{CaCO}_{3}$ microparticles were mixed with $1 \mathrm{~mL}$ copper (II) perchlorate solution (1M) and incubated for 1, 3 and 6 hours. The PPy loaded $\mathrm{CaCO}_{3}$ microparticles were washed consequently twice following the sequence of 2propanol, ethanol and water by centrifugation (2000 rpm, $1 \mathrm{~min}$ ) and re-dispersion cycles. Subsequently, the $\mathrm{CaCO}_{3}$ template was removed by addition of $1 \mathrm{~mL}$ EDTA solution $(0.2 \mathrm{M})$ and incubated for 1 hour at room temperature. Finally, pure PPyMPs were washed twice with water by centrifugation (2000 rpm, $1 \mathrm{~min}$ ) and re-dispersion cycles.

Optical microscopy images were recorded using a Nikon ECLIPSE Ti (Nikon, Japan). Images were captured by using a NIS-Elements AR (Version 4.1, Nikon, Japan). Scanning electron microscopy (SEM) images of the microparticle film were recorded with PHENOM PRO (FEI, Netherlands). High resolution SME images of the microparticles were recorded with LEO 1550 Gemini, (Zeiss, Germany). Microparticle samples suspended in milli-Q water were applied onto a flat silicon surface, air dried at ambient temperature, and coated with platinum.

All electrochemical experiments and simulations were performed with a PGSTAT30 potentiostat (Autolab, Nertherland) under GPES software control employing a conventional three-electrode electrochemical cell. A glassy carbon electrode (GCE, $3 \mathrm{~mm}$ diameter, surface area $0.0707 \mathrm{~cm}^{2}$ ) was used as the working electrode, a platinum wire as the auxiliary electrode, and a silver/silver chloride as the reference electrode $(3 \mathrm{M} \mathrm{KCl})$ in aqueous media in all experiments. Prior to use the working electrode was successively polished with 1.0, 0.3 and 0.05 $\mu \mathrm{m}$ alumina powders and sonicated in water for $10 \mathrm{~min}$ after each polishing step. Finally, the electrode was washed with ethanol and then dried with a high purity argon stream. The GCE were modified with PPyMPs by drop-casting of aqueous solutions followed by curing at 60 ${ }^{\circ} \mathrm{C}$ for $30 \mathrm{~min}$. Before the actual measurements all films were equilibrated with cyclic voltammetry (from $0 \mathrm{~V}$ up to $0.4 \mathrm{~V}, 100 \mathrm{mV} \mathrm{s}^{-1}$ ) until the stable voltammograms were obtained.

\section{Acknowledgements}

This work was supported by the Scholarship for an overseas thesis research study supported by Graduate School Prince of Songkla University. Finacial support from the Higher Education Research Promotion and National Research University Project of Thailand (NRU); Office of higher Education Commission; the Center of Excellence for Innovation in Chemistry (PERCH-CIC), Commission on Higher Education, Ministry of Education, Thailand.

\section{References}

[1] aA. J. Heeger, Angewandte Chemie-International Edition 2001, 40, 2591-2611; bR. Menon, C. O. Yoon, D. Moses, A. J. Heeger, Metal-insulator transition in doped conducting polymers, 2nd edition ed., Marcel Dekker, New York, 1998.

[2] aF. Jaouen, E. Proietti, M. Lefevre, R. Chenitz, J. P. Dodelet, G. Wu, H. T. Chung, C. M. Johnston, P. Zelenay, Energy \& Environmental Science 2011, 4, 114-130; bE. Proietti, F. Jaouen, M. Lefèvre, N. Larouche, J. Tian, J. Herranz, J.-P. Dodelet, Nature Communications 2011, 2, 416.

[3] aY. Cao, T. E. Mallouk, Chemistry of Materials 2008, 20, 5260-5265; bJ. Jang, Advances in Polymer Science 2006, 199, 189-259; cG. F. Li, C. Martinez, S. Semancik, Journal of the American Chemical Society 2005, 127, 4903-4909; dC. R. Martin, L. S. Vandyke, Z. H. Cai, W. B. Liang, Journal of the American Chemical Society 1990, 112, 8976-8977; eR. M. Penner, C. R. Martin, Journal of The Electrochemical Society 1986, 133, 2206-2207.

[4] aM. Y. Bai, Y. J. Cheng, S. A. Wickline, Y. N. Xia, Small 2009, 5, 1747-1752; bC. H. Chang, P. S. Son, J.-A. Yoon, S.-H. Choi, Journal of Nanomaterials 2010, Article ID 168025, 1-6; cM. G. Han, S. H. Foulger, Chemical Communications 2004, 19, 2154-2155; dL. Hao, C. Zhu, C. Chen, P. Kang, Y. Hu, W. Fan, Z. Chen, Synthetic Metals 2003, 139, 391-396; eX. Liu, H. Wu, F. Ren, G. Qiu, M. Tang, Materials Chemistry and Physics 2008, 109, 5-9; fM. Marti, G. Fabregat, F. Estrany, C. Aleman, E. Armelin, Journal of Materials Chemistry 2010, 20, 10652-10660; gM. Mazur, Langmuir 2008, 24, 1041410420; hA. B. Slimane, K. Boukerma, M. Chabut, M. M. Chehimi, Colloids and Surfaces A: Physicochemical and Engineering Aspects 2004, 240, 45-53; iF. Yang, Y. Chu, S. Ma, Y. Zhang, J. Liu, Journal of Colloid and Interface Science 2006, 301, 470-478; jX. Yang , Y. Lu, Polymer 2005, 46, 5324-5328. 
[5] aC. C. Han, M. Y. Bai, K. F. Yang, Y. S. Lee, C. W. Lin, Journal of Materials Chemistry 2008, 18, 3918-3925; bE. Jin, N. Liu, X. F. Lu, W. J. Zhang, Chemistry Letters 2007, 36, 1288-1289; cN. P. Tavandashti, M. Ghorbani, A. Shojaei, Polymer 2013, 54, 5586-5594; dE. C. Venancio, P. C. Wang, A. G. MacDiarmid, Synthetic Metals 2006, 156, 357-369; eZ. Wei, M. Wan, Advanced Materials 2002, 14, 1314-1317; fH. M. Zhang, Y. P. Li, X. H. Wang, J. Li, F. S. Wang, Polymer 2011, 52, 4246-4252; gL. J. Zhang, M. Wang, Y. Wei, Macromolecular Rapid Communications 2006, 27, 888-893.

[6] R. Wannapob, M. Y. Vagin, I. Jeerapan, W. C. Mak, Langmuir 2015, 31, 11904-11913.

[7] M. Y. Vagin, I. Jeerapan, R. Wannapob, P. Thavarungkul, P. Kanatharana, N. Anwar, T. McCormac, M. Eriksson, A. P. F. Turner, E. W. H. Jager, W. C. Mak, Electrochimica Acta 2016, 190, 495-503.

[8] aY. Gu, H. Wu, Z. Xiong, W. Al Abdulla, X. S. Zhao, Journal of Materials Chemistry A 2014, 2, 451-459; bJ. J. Yoo, K. Balakrsihnan, J. S. Huang, V. Meunier, B. G. Sumper, A. Srivastava, M. Conway, A. L. M. Reddy, J. Yu, R. Vajtai, P. M. Ajayan, Nano Letters 2011, 11, 14231427.

[9] aB. Babakhani, D. G. Ivey, Electrochimica Acta 2011, 56, 4753-4762; bX. Lang, A. Hirata, T. Fujita, M. Chen, Nature Nanotechnology 2011, 6, 232-236.

[10] aP. A. Christensen, A. Hamnett, Electrochimica Acta 1991, 36, 1263-1286; bA. A. Pud, Synthetic Metals 1994, 66, 1-18.

[11] aB. Yue, C. Wang, X. Ding, G. G. Wallace, Electrochimica Acta 2012, 68, 18-24; bJ. Xu, D. Wang, L. Fan, Y. Yuan, W. Wei, R. Liu, S. Gu, W. Xu, Organic Electronics 2015, 26, 292-299; cC. Shen, Y. Sun, W. Yao, Y. Lu, Polymer 2014, 55, 2817-2824; dX. Li, I. Zhitomirsky, Journal of Power Sources 2013, 221, 49-56; eM. S. Kumar, D. K. Bhat, Journal of Applied Polymer Science 2009, 114, 2445-2454; fA. Faye, G. Dione, M. M. Dieng, J. J. Aaron, H. Cachet, C. Cachet, Journal of Applied Electrochemistry 2010, 40, 1925-1931; gH. Du, Y. Xie, C. Xia, W. Wang, F. Tian, Y. Zhou, Materials Letters 2014, 132, 417-420; hM. Ates, M. A. Serin, I. Ekmen, Y. N. Ertas, Polymer Bulletin 2015, 72, 25732589. 